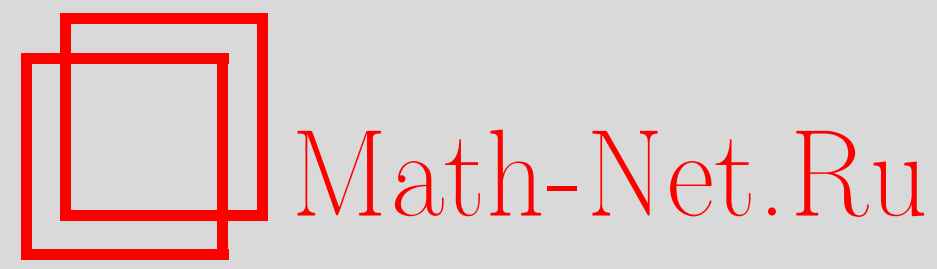

Н. В. Лазакович, С. П. Сташуленок, Т. В. Стемковская, Ассоциированные решения уравнений в дифференциалах в прямом произведении алгебр обобщенных случайных процессов, Теория вероятн. и ее примен., 1998, том 43, выпуск 2, 272-293

DOI: https://doi.org/10.4213/tvp1465

Использование Общероссийского математического портала Math-Net.Ru подразумевает, что вы прочитали и согласны с пользовательским соглашением http://www. mathnet.ru/rus/agreement

Параметры загрузки:

IP: 54.224 .187 .69

26 апреля 2023 г., $12: 37: 21$

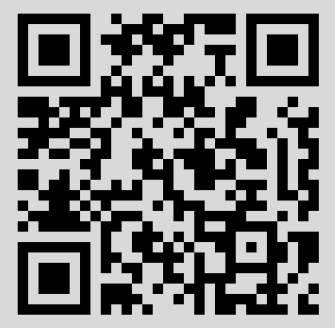


(C) 1998 г. ЛАЗАКОВИЧ Н. В.*, СТАШУЛЕНОК С. П.*, СТЕМКОВСКАЯ Т. В.*

\section{АССОЦИИРОВАННЫЕ РЕШЕНИЯ УРАВНЕНИЙ В ДИФФЕРЕНЦИАЛАХ В ПРЯМОМ ПРОИЗВЕДЕНИИ АЛГЕБР ОБОБЩЕННЫХ СЛУЧАЙНЫХ ПРОЩЕССОВ ${ }^{1)}$}

В прямом произведении алгебр обобщенных случайных процессов рассматриваются уравнения в дифференциалах и исследуются их ассоциированные решения. Доказывается, что в одних случаях они являются решениями соответствующих стохастических дифференциальных уравнений Ито, в других - решениями уравнений Стратоновича. В терминах алгебр обобщенных случайных процессов дается описание областей применимости стохастических дифференциальных уравнений Ито и Стратоновича.

Ключевые слова и фразы: стохастические дифференциальные уравнения Ито и Стратоновича, прямое произведение алгебр обобшенных случайных процессов, обобщенные дифференциалы Ито и Стратоновича, ассоциированные решения уравнений в дифференциалах.

Известно, что при изучении дифференциальных уравнений со случайными функциями различают два случая. В первом из них случайные функции, входящие в дифференциальное уравнение, достаточно регулярны, и большинство вопросов, связанных с исследованием свойств решения уравнения, можно решить классическими методами теории обыкновенных дифференциальных уравнений.

Во втором случае приходится рассматривать дифференциальные уравнения, содержашие обобщенные случайные процессы типа «белого шума». K таким уравнениям неприменимы классические методы. Для них разработана специальная теория стохастических дифференциальных уравнений. Однако и в этом случае многие авторы не отказались от попыток исследования решений классическими методами, правда, на аппроксимационном уровне. В работах [1]-[6] исследуется задача аппроксимации решений стохастических цифференциальных уравнений

*Белорусский государственный университет, механико-математический факультет, пр. Ф. Скорины, 4, 220080 Минск, Республика Беларусь.

1) Работа выполнена при поддержке Фонда фундаментальных исследований Республики Беларусь и Международной Соросовской программы образования в области точных наук. 
решениями либо конечно-разностных, либо обыкновенных дифференциальных уравнений.

Следует отметить, что решениями конечно-разностных уравнений, как правило, «приближаются» решения стохастических дифференциальных уравнений Ито, а решениями обыкновенных дифференциальных уравнений - решения стохастических дифференциальных уравнений Стратоновича. И только для обыкновенных дифференциальных уравнений с запаздыванием при некоторых аппроксимациях броуновского движения с помощью полигонального метода [7], [8] можно добиться и аппроксимации решения стохастического дифференциального уравнения Ито [9].

Естественно, при этом встает задача разделения областей применимости стохастических дифференциальных уравнений Ито и Стратоновича $[10]$.

Настоящая статья - продолжение работы [11]. Она состоит из двух частей. В первой установлено, что решения многомерного стохастического дифференциального уравнения как Ито, так и Стратоновича, могут быть аппроксимированы решениями соответствующего конечноразностного уравнения с осреднением. Рассмотрен также смешанный случай и даны описания «областей» Ито и Стратоновича.

Во второй части приводятся теоремы существования и единственности решений уравнений в дифференциалах в прямом произведении алгебр обобщенных случайных процессов и исследуются ассощиированные решения этих уравнений.

\section{Введение}

Приведем некоторые понятия из работы [11], которые мы будем использовать в дальнейшем.

Пусть $(\Omega, \mathscr{A}, \mathbf{P})$ - полное вероятностное пространство, $T=$ $[0, a] \subset \mathbf{R},\left\{\mathscr{F}_{t}\right\}_{t \in T}-$ стандартный поток $\sigma$-алгебр, $\mathscr{F}_{a} \subset \mathscr{A}$.

Рассмотрим множество последовательностей функций

$$
f_{n}(t, \omega): \mathbf{N} \times T \times \Omega \rightarrow \mathbf{R}
$$

таких, что $f_{n}(t, \omega)$ - случайная величина на $(\Omega, \mathscr{A}, \mathbf{P})$ для любого $t \in T$ и $n \in \mathrm{N}$.

Элементы $\boldsymbol{F}=\left(f_{n}\right)$ и $G=\left(g_{n}\right)$ данного множества назовем эквивалентными, если сушествует такое $n_{0}$, что $f_{n}(t, \omega)=g_{n}(t, \omega)$ для любых $t \in T$, почти всех $\omega \in \Omega$ и $\forall n>n_{0}$.

Множество классов эквивалентных последовательностей такого вида обозначим $\mathscr{G}_{L}(T, \Omega)$, если для любых $n \in \mathbf{N}$ функции $f_{n}(t, \omega)$ являются бесконечно дифференцируемыми на $T$ в смысле $L^{p}(\Omega, \mathscr{A}, \mathbf{P})$ для всех $p \geqslant 1$, и $\mathscr{G}(T, \Omega)$, если для любых $n \in \mathbf{N} f_{n}(t, \omega) \in C^{\infty}(T)$ для почти всех $\omega \in \Omega$. 
Несложно видеть, что $\mathscr{G}_{L}(T, \Omega)$ и $\mathscr{G}(T, \Omega)$ являются алгебрами с покоординатными операциями сложения и умножения. Далее, пусть $\widetilde{T}=\left\{\tilde{t}=\left[\left(t_{n}\right)\right] \in \widetilde{\mathbf{R}}: \forall\left(t_{n}\right) \in \tilde{t}, 0 \leqslant t_{n} \leqslant a, n=1,2, \ldots\right\}$, где $\widetilde{\mathbf{R}}-$ расширенная прямая из [12]. Через $\mathscr{G}_{L}(\widetilde{T}, \Omega)($ соответственно, $(\mathscr{G}(\widetilde{T}, \Omega))$ обозначим алгебру обобпенных случайных процессов $\widetilde{F}(\tilde{t}, \omega)$ вида:

$$
\widetilde{F}(\tilde{t}, \omega)=\left[\left(f_{n}\left(t_{n}, \omega\right)\right)\right],
$$

где $\tilde{t}=\left[\left(t_{n}\right)\right] \in \widetilde{T}$, а $\left[\left(f_{n}(t, \omega)\right)\right] \in \mathscr{G}_{L}(T, \Omega)$ (соответственно, $\left[\left(f_{n}(t, \omega)\right)\right] \in$ $\mathscr{G}(T, \Omega))$.

Введем на $\widetilde{\mathbf{R}}$ множество

$$
H=\left\{\widetilde{h}=\left[\left(h_{n}\right)\right] \in \widetilde{\mathbf{R}}_{+}|\widetilde{0}| \lim _{n \rightarrow \infty} h_{n}=0\right\}
$$

и для $\forall \widetilde{F}=\left[\left(f_{n}\right)\right]$ из $\mathscr{G}_{L}(\widetilde{T}, \Omega)$ или $\mathscr{G}(\widetilde{T}, \Omega)$ положим по определению

$$
d_{\tilde{h}} \tilde{F}(\tilde{t}, \omega)=\left[\left(f_{n}\left(t+h_{n}, \omega\right)-f_{n}(t, \omega)\right)\right],
$$

где $\tilde{t}=[(t)] \in \widetilde{T}, \widetilde{h} \in H, \tilde{t}+\widetilde{h} \in \widetilde{T}$.

Выделим в $H$ подмножества

$$
\begin{aligned}
& I=\left\{\widetilde{h} \in H \mid n^{-1}=o\left(h_{n}\right) \forall\left(h_{n}\right) \in \widetilde{h}\right\}, \\
& S=\left\{\widetilde{h} \in H \mid h_{n}=o\left(n^{-1}\right) \forall\left(h_{n}\right) \in \widetilde{h}\right\} .
\end{aligned}
$$

Напомним, что $d_{\widetilde{h}} \widetilde{F}, \widetilde{h} \in S$, называется обобщенным дифференциалом Стратоновича, а $d_{\widetilde{h}} \widetilde{F}, \widetilde{h} \in I,-$ обобщенным дифференциалом Ито.

Через $\mathscr{G}_{L}(T, \Omega)^{r}, \mathscr{G}_{L}(\tilde{T}, \Omega)^{r}, \mathscr{G}(T, \Omega)^{r}, \mathscr{G}(\widetilde{T}, \Omega)^{r}$ обозначим прямое произведение алгебр $\mathscr{G}_{L}(T, \Omega), \mathscr{G}_{L}(\widetilde{T}, \Omega), \mathscr{G}(T, \Omega)$ и $\mathscr{G}(\widetilde{T}, \Omega)$ соответственно, $r \in \mathbf{N} ; B(t, \omega)=\left(B^{1}(t, \omega), \ldots, B^{T}(t, \omega)\right), t \in T, \omega \in \Omega$, есть $r$-мерный стандартный процесс $\mathscr{F}_{t}$-броуновского движения $[13$, c. $48-49]$.

Будем говорить, что обобщенный случайный процесс

$$
F(t, \omega)=\left[\left(f_{n}(t, \omega)\right)\right] \in \mathscr{G}(T, \Omega) \quad\left(\mathscr{G}_{L}(T, \Omega)\right)
$$

aсcочиирует классический случайный процесс, если для любых представителей $\left(f_{n}\right) \in F$ последовательность $f_{n}(t, \omega)$ сходится при $n \rightarrow \infty$ к данному процессу почти всюду (в $\left.L^{2}(\Omega, \mathscr{A}, \mathbf{P})\right)$ и равномерно по $t \in T$.

В многомерном случае рассматриваем покоординатное ассоциирование.

Обобщенным случайным процессом броуновского движения назовем элемент пространства алгебр $\mathscr{G}_{L}(T, \Omega)^{r}\left(\mathscr{G}(T, \Omega)^{r}\right)$, ассоциируюший $r$ мерный процесс $\mathscr{F}_{t}$-броуновского движения $B$. 


\section{1. Конечно-разностные аппроксимации решений стохастических дифференциальных уравнений}

В этом разделе исследуется задача аппроксимации решения системы стохастических дифференциальных уравнений решениями конечноразностных уравнений с осреднением. Аналогичные аппроксимационные подходы в детерминистическом случае применялись в работах [14], [15]. Доказывается, что как решения стохастического дифференциального уравнения Ито, так и решения Стратоновича могут быть аппроксимированы решениями конечно-разностного уравнения. Параллельно, в терминах множеств $S$ и $I$ дается описание «областей» Стратоновича и Ито.

\section{1. Аппроксимация решений стохастических дифференци-} альных уравнений Ито решениями конечно-разностных уравнений с запаздыванием. Рассмотрим систему стохастических дифференциальных уравнений

$$
\begin{aligned}
X^{i}(t, \omega)= & x^{i}+\sum_{j=1}^{r}(I) \int_{0}^{t} \sigma^{i j}(X(s, \omega)) d B^{j}(s, \omega) \\
& +\int_{0}^{t} a^{i}(X(s, \omega)) d s, \quad i=\overline{1, d}, t \in T
\end{aligned}
$$

где $X(t, \omega)=\left(X^{1}(t, \omega), \ldots, X^{d}(t, \omega)\right), x=\left(x^{1}, \ldots, x^{d}\right) \in \mathbf{R}^{d}, a^{i} \in C_{B}^{\mathbf{1}}\left(\mathbf{R}^{d}\right)$, $\sigma^{i j} \in C_{B}^{2}\left(\mathbf{R}^{d}\right), i=\overline{1, d}, j=\overline{1, r}$, стохастический интеграл в правой части (1.1) - стохастический интеграл Ито $\left[13\right.$, с. 53], $C_{B}^{l}\left(\mathbf{R}^{d}\right), l=1,2,-$ множество действительных $l$ раз непрерывно дифференцируемых на $\mathbf{R}^{d}$ функций, ограниченных вместе со своими частными производными до порядка $l$ включительно.

Существование и единственность решения системы (1.1) доказаны в $[13$, с. 233].

Пусть

$$
\begin{aligned}
& B_{n}^{j}(t, \omega)=\left(B^{j} * \rho_{n}\right)(t, \omega)=\int_{0}^{1 / n} B^{j}(t+s, \omega) \rho_{n}(s) d s, \quad j=\overline{1, r} \\
& \rho_{n}(s)=n \rho(n s), \quad s \in \mathbf{R}, n \in \mathbf{N}, \rho \in C^{\infty}(\mathbf{R}), \rho \geqslant 0, \operatorname{supp} \rho \subset[0,1] \\
& \int_{0}^{1} \rho(s) d s=1 ; \quad \sigma_{n}^{i j}(u)=\left(\sigma^{i j} * \bar{\rho}_{n}\right)(u), \quad a_{n}^{i}(u)=\left(a^{i} * \bar{\rho}_{n}\right)(u), \\
& u \in \mathbf{R}^{d}, \quad i=\overline{1, d}, \quad j=\overline{1, r}, \quad \bar{\rho}_{n}(u)=n^{d} \bar{\rho}(n u), \quad \bar{\rho} \in C^{\infty}\left(\mathbf{R}^{d}\right) \\
& \operatorname{supp} \bar{\rho} \subset[0,1]^{d}, \quad \bar{\rho} \geqslant 0, \quad \int_{[0,1]^{d}} \bar{\rho}(u) d u=1,
\end{aligned}
$$

$\|\cdot\|$ - евклидова норма в $\mathbf{R}^{d}$. 
Исследуем задачу апшроксимации решения системы (1.1) решениями конечно-разностных уравнений с запаздыванием

$$
\left\{\begin{aligned}
X_{n}^{i}\left(t+h_{n}, \omega\right)-X_{n}^{i}(t, \omega) & \\
=\sum_{j=1}^{r} \sigma_{n}^{i j}\left(X_{n}\left(t-h_{n}, \omega\right)\right) & {\left[B_{n}^{j}\left(t+h_{n}, \omega\right)-B_{n}^{j}(t, \omega)\right] } \\
\quad+a_{n}^{i}\left(X_{n}(t, \omega)\right) h_{n}, & t \in T, \\
\left.X_{n}^{i}\right|_{\left[-h_{n}, h_{n}\right)}=X_{0 n}^{i}(t, \omega), & i=\overline{1, d}
\end{aligned}\right.
$$

где случайный процесс $X_{0 n}(t, \omega)=\left(X_{0 n}^{1}(t, \omega), \ldots, X_{0 n}^{d}(t, \omega)\right)$ согласован с потоком $\sigma$-алгебр $\mathscr{F}_{t+1 / n}, t \in\left[0, h_{n}\right)$, и принадлежит классу $C^{\infty}$ в смысле $L^{2}(\Omega, \mathscr{A}, \mathbf{P})$ (по определению полагаем, что $X_{n}^{i}(t, \omega)=X_{n}^{i}(0, \omega), t<0$ ).

Теорема 1. Пусть $X(t, \omega), X_{n}(t, \omega), t \in T, \omega \in \Omega, \quad$ - решения задач (1.1) и (1.3) соответственно, $\sigma^{i j} \in C_{B}^{2}\left(\mathbf{R}^{d}\right), a^{i} \in C_{B}^{1}\left(\mathbf{R}^{d}\right), i=\overline{1, d}$, $i=\overline{1, r}$. Toada

$$
\begin{aligned}
& \sup _{t \in T} \mathbf{E}\left\|X(t, \omega)-X_{n}(t, \omega)\right\|^{2} \\
& \quad \leqslant C \sup _{t \in\left[0, h_{n}\right)} \mathbf{E}\left\|x-X_{0 n}(t, \omega)\right\|^{2}+C h_{n}+\frac{C}{n h_{n}}, \quad \frac{1}{n}<h_{n} .
\end{aligned}
$$

Д о к а з а т е л ь с т в о. Пусть $\tau$ - произвольная точка из $T$. Тогда найдутся такие $t_{\tau} \in\left[0, h_{n}\right)$ и $k_{\tau}=0,1,2, \ldots$, что $\tau=t_{\tau}+k_{\tau} h_{n}$.

Поэтому решение задачи Коши (1.1) можно записать в виде

$$
\begin{aligned}
X_{n}^{i}(\tau, \omega)= & X_{0 n}^{i}\left(t_{\tau}, \omega\right)+\sum_{j=1}^{r} \sum_{l=1}^{k_{\tau}} \sigma_{n}^{i j}\left(X_{n}\left(t_{\tau}+(l-2) h_{n}, \omega\right)\right) \\
& \times\left[B_{n}^{j}\left(t_{\tau}+l h_{n}, \omega\right)-B_{n}^{j}\left(t_{\tau}+(l-1) h_{n}, \omega\right)\right] \\
& +\sum_{l=1}^{k_{\tau}} a_{n}^{i}\left(X_{n}\left(t_{\tau}+(l-1) h_{n}, \omega\right)\right) h_{n}, \quad i=\overline{1, d}
\end{aligned}
$$

Тогда

$$
\begin{array}{r}
\left\|X(\tau, \omega)-X_{n}(\tau, \omega)\right\| \leqslant\left\|x-X_{0 n}\left(t_{\tau}, \omega\right)\right\| \\
+\| \sum_{j=1}^{r}(I) \int_{0}^{\tau} \sigma^{j}(X(s, \omega)) d B^{j}(s, \omega) \\
-\sum_{j=1}^{r} \sum_{l=1}^{k_{\tau}} \sigma_{n}^{j}\left(X_{n}\left(t_{\tau}+(l-2) h_{n}, \omega\right)\right)
\end{array}
$$




$$
\begin{gathered}
\times\left[B_{n}^{j}\left(t_{\tau}+l h_{n}, \omega\right)-B_{n}^{j}\left(t_{\tau}+(l-1) h_{n}, \omega\right)\right] \| \\
+\left\|\int_{0}^{\tau} a(X(s, \omega)) d s-\sum_{l=1}^{k_{\tau}} a_{n}\left(X_{n}\left(t_{\tau}+(l-1) h_{n}, \omega\right)\right) h_{n}\right\| \\
=I_{1}(\tau, \omega)+I_{2}(\tau, \omega)+I_{3}(\tau, \omega) .
\end{gathered}
$$

Рассмотрим $I_{2}(\tau, \omega)$. Несложно убедиться в том, что

$$
I_{2}(\tau, \omega) \leqslant I_{21}(\tau, \omega)+I_{22}(\tau, \omega)+I_{23}(\tau, \omega)+I_{24}(\tau, \omega)+I_{25}(\tau, \omega)
$$

где

$$
\begin{aligned}
I_{21}(\tau, \omega)=\| & \sum_{j=1}^{r}(I) \int_{0}^{\tau} \sigma^{j}(X(s, \omega)) d B^{j}(s, \omega) \\
& -\sum_{j=1}^{r}(I) \int_{0}^{\tau} \sigma_{n}^{j}(X(s, \omega)) d B^{j}(s, \omega) \| \\
I_{22}(\tau, \omega)=\| & \sum_{j=1}^{r}(I) \int_{0}^{\tau} \sigma_{n}^{j}(X(s, \omega)) d B^{j}(s, \omega) \\
& -\sum_{j=1}^{r} \sigma_{n}^{j}(X(0, \omega))\left[B^{j}\left(t_{\tau}, \omega\right)-B^{j}(0, \omega)\right] \\
& -\sum_{j=1}^{r} \sum_{l=1}^{k_{\tau}} \sigma_{n}^{j}\left(X\left(t_{\tau}+(l-2) h_{n}, \omega\right)\right) \\
& \times\left[B^{j}\left(t_{\tau}+l h_{n}, \omega\right)-B^{j}\left(t_{\tau}+(l-1) h_{n}, \omega\right)\right] \| \\
& \quad-\left[B_{j=1}^{j}\left(t_{\tau}+l h_{n}, \omega\right)-B^{j}\left(t_{\tau}+(l-1) h_{n}, \omega\right)\right] \| \\
& \times\left[B^{j}\left(t_{\tau}+l h_{n}, \omega\right)-B^{j}\left(t_{\tau}+(l-1) h_{n}, \omega\right)\right] \\
I_{23}(\tau, \omega)= & \sum_{j=1}^{r} \sigma_{n}^{j}(X(0, \omega))\left[B^{j}\left(t_{\tau}, \omega\right)-B^{j}(0, \omega)\right] \| \\
\| & \sum_{j=1}^{r} \sum_{l=1}^{k_{\tau}} \sigma_{n}^{j}\left(X\left(t_{\tau}+(l-2) h_{n}, \omega\right)\right)
\end{aligned}
$$




$$
\begin{aligned}
I_{25}(\tau, \omega)=\| & \sum_{j=1}^{r} \sum_{l=1}^{k_{\tau}} \sigma_{n}^{j}\left(X_{n}\left(t_{\tau}+(l-2) h_{n}, \omega\right)\right) \\
& \times\left[B^{j}\left(t_{\tau}+l h_{n}, \omega\right)-B^{j}\left(t_{\tau}+(l-1) h_{n}, \omega\right)\right] \\
& -\sum_{j=1}^{r} \sum_{l=1}^{k_{\tau}} \sigma_{n}^{j}\left(X_{n}\left(t_{\tau}+(l-2) h_{n}, \omega\right)\right) \\
& \times\left[B_{n}^{j}\left(t_{\tau}+l h_{n}, \omega\right)-B_{n}^{j}\left(t_{\tau}+(l-1) h_{n}, \omega\right)\right] \|
\end{aligned}
$$

Из представления $\sigma_{n}^{i j}$, неравенства Гёльдера и теоремы Лагранжа вытекает, что

$$
\mathbf{E}\left[I_{21}(\tau, \omega)\right]^{2} \leqslant C n^{-2}
$$

Пусть $\bar{X}(t, \omega)=X\left(t_{\tau}+(l-2) h_{n}, \omega\right)$ для $t \in\left[t_{\tau}+(l-1) h_{n}, t_{\tau}+l h_{n}\right)$, $l=\overline{2, k_{\tau}}$ и $\bar{X}(t, \omega)=X(0, \omega)$ для $t \in\left[0, t_{\tau}+h_{n}\right)$. Тогда

$$
\begin{aligned}
\sigma_{n}^{i j}(X(0, \omega))\left[B^{j}\left(t_{\tau}, \omega\right)-B^{j}(0, \omega)\right]-\sum_{l=1}^{k_{\tau}} \sigma_{n}^{i j}\left(X\left(t_{\tau}+(l-2) h_{n}, \omega\right)\right) \\
\quad \times\left[B^{j}\left(t_{\tau}+l h_{n}, \omega\right)-B^{j}\left(t_{\tau}+(l-1) h_{n}, \omega\right)\right] \\
=(I) \int_{0}^{\tau} \sigma_{n}^{i j}(X(s, \omega)) d B^{j}(s, \omega) .
\end{aligned}
$$

Поэтому, используя свойства интеграла Ито, теорему Лагранжа, то, что $\sigma^{i j} \in C_{B}^{2}\left(\mathbf{R}^{d}\right)$, соотношение (1.7), а также уравнение (1.1), получим

$$
\begin{gathered}
\mathbf{E}\left[I_{22}(\tau, \omega)\right]^{2} \leqslant C h_{n}, \quad \mathbf{E}\left[I_{23}(\tau, \omega)\right]^{2} \leqslant C h_{n}, \\
\mathbf{E}\left[I_{24}(\tau, \omega)\right]^{2} \leqslant \sum_{i=1}^{d} r \sum_{j=1}^{r} \sum_{l=1}^{k_{\tau}} \mathbf{E}\left\{\left[\sigma_{n}^{i j}\left(X\left(t_{\tau}+(l-2) h_{n}, \omega\right)\right)\right.\right. \\
\left.\quad-\sigma_{n}^{i j}\left(X_{n}\left(t_{\tau}+(l-2) h_{n}, \omega\right)\right)\right] \\
\left.\times\left[B^{j}\left(t_{\tau}+l h_{n}, \omega\right)-B^{j}\left(t_{\tau}+(l-1) h_{n}, \omega\right)\right]\right\}^{2} \\
\leqslant C h_{n} \sum_{l=1}^{k_{\tau}} \mathbf{E} \| X\left(t_{\tau}+(l-2) h_{n}, \omega\right) \\
-X_{n}\left(t_{\tau}+(l-2) h_{n}, \omega\right) \|^{2}
\end{gathered}
$$

Учитывая то, что математические ожидания удвоенных произведений вида

$2\left[B^{j}\left(t_{\tau}+l h_{n}, \omega\right)-B_{n}^{j}\left(t_{\tau}+l h_{n}, \omega\right)\right]\left[B^{j}\left(t_{\tau}+m h_{n}, \omega\right)-B_{n}^{j}\left(t_{\tau}+m h_{n}, \omega\right)\right]$ 
равны нулю для $l<m$, имеем

$$
\mathbf{E}\left[I_{25}(\tau, \omega)\right]^{2} \leqslant C \sum_{l=1}^{k_{\tau}} \frac{1}{n} \int_{0}^{1} y \rho_{n}^{2}(y) d y \leqslant \frac{C k_{a}}{n} .
$$

В итоге из соотношений $(1.5),(1.6),(1.8)-(1.10)$ для $I_{2}(\tau, \omega)$ получим следующую ощенку сверху:

$$
\begin{aligned}
& \mathbf{E}\left[I_{2}(\tau, \omega)\right]^{2} \leqslant \frac{C}{n^{2}}+\frac{C k_{a}}{n} \\
& \quad+C h_{n} \sum_{l=2}^{k_{\tau}} \mathbf{E}\left\|X\left(t_{\tau}+(l-2) h_{n}, \omega\right)-X_{n}\left(t_{\tau}+(l-2) h_{n}, \omega\right)\right\|^{2} .
\end{aligned}
$$

Исследуем $I_{3}(\tau, \omega)$. Используя оценки (1.8), неравенство Гёльдера, теоремы Лагранжа и Фубини, получаем:

$$
\begin{aligned}
& \mathbf{E}\left[I_{3}(\tau, \omega)\right]^{2} \leqslant C h_{n} \\
& \quad+C h_{n} \sum_{l=1}^{k_{\tau}} \mathbf{E}\left\|X\left(t_{\tau}+(l-1) h_{n}, \omega\right)-X_{n}\left(t_{\tau}+(l-1) h_{n}, \omega\right)\right\|^{2} .
\end{aligned}
$$

Таким образом, из соотношений $(1.4),(1.11),(1.12)$ следует

$$
\begin{aligned}
& \mathbf{E}\left\|X(\tau, \omega)-X_{n}(\tau, \omega)\right\|^{2} \leqslant C \sup _{t \in\left[-h_{n}, h_{n}\right)} \mathbf{E}\left\|x-X_{0 n}(t, \omega)\right\|^{2} \\
& \quad+\frac{C}{n^{2}}+\frac{C k_{a}}{n}+C h_{n}+C h_{n} \sum_{l=1}^{k_{\tau}} \mathbf{E} \| X\left(t_{\tau}+(l-1) h_{n}, \omega\right) \\
& \quad-X_{n}\left(t_{\tau}+(l-1) h_{n}, \omega\right) \|^{2} .
\end{aligned}
$$

Методом математической индукции можно доказать, что,

$$
\text { если } x_{k} \leqslant a+b \sum_{l=1}^{k} x_{l-1}, \forall k \in \mathbf{N}, \text { mo } x_{k} \leqslant(b+1)^{k-1}\left(a+b x_{0}\right) .
$$

Воспользовавшись утверждением (1.14), получаем, что из неравенства (1.13) следует утверждение теоремы.

Следствие 1. Пусть $\sigma^{i j} \in C_{B}^{2}\left(\mathbf{R}^{d}\right), a^{i} \in C_{B}^{1}\left(\mathbf{R}^{d}\right), i=\overline{1, d}, j=\overline{1, r}$, $u n \rightarrow \infty, h_{n} \rightarrow 0 \max$, umo

$$
\begin{gathered}
n^{-1}=o\left(h_{n}\right) \\
\sup _{t \in\left[0, h_{n}\right)} \mathbf{E}\left\|x-X_{0 n}(t, \omega)\right\|^{2} \longrightarrow 0
\end{gathered}
$$


Tогдa

$$
\sup _{t \in T} \mathrm{E}\left\|X(t, \omega)-X_{n}(t, \omega)\right\|^{2} \longrightarrow 0,
$$

где $X(t, \omega)$ и $X_{n}(t, \omega)$ - решения задач (1.1) и (1.3) соответственно.

3 а м е ч а н и е 1. Несложно видеть, что условие (1.15) эквивалентно тому, что $\widetilde{h}=\left[\left(h_{n}\right)\right] \in I$.

3 а м е ч а н и е2. Результаты следствия 1 остаются справедливыми и для запаздывания $1 / n$.

\section{2. Аппроксимация решений стохастических дифференци-} альных уравнений Ито решениями конечно-разностных уравнений. Исследуем теперь задачу аппроксимации решений системы (1.1) решениями конечно-разностных уравнений с осреднением

$$
\left\{\begin{array}{l}
Y_{n}^{i}\left(t+h_{n}, \omega\right)-Y_{n}^{i}(t, \omega) \\
=\sum_{j=1}^{r} \sigma_{n}^{i j}\left(Y_{n}(t, \omega)\right)\left[B_{n}^{j}\left(t+h_{n}, \omega\right)-B_{n}^{j}(t, \omega)\right] \\
\quad+a_{n}^{i}\left(Y_{n}(t, \omega)\right) h_{n}, \\
\left.Y_{n}^{i}\right|_{\left[0, h_{n}\right)}=X_{0 n}^{i}(t, \omega), \quad i=\overline{1, d}, j=\overline{1, r}, t \in T,
\end{array}\right.
$$

где случайный процесс

$$
X_{0 n}(t, \omega)=\left(X_{0 n}^{1}(t, \omega), \ldots, X_{0 n}^{d}(t, \omega)\right)
$$

согласован с потоком $\sigma$-алгебр $\mathscr{F}_{t+1 / n}, t \in\left[0, h_{n}\right)$, и принадлежит классу $C^{\infty}$ в смысле $L^{2}(\Omega, \mathscr{A}, \mathbf{P})$.

Теорема 2. Пусть $\sigma^{i j} \in C_{B}^{2}\left(\mathbf{R}^{d}\right), a^{i} \in C_{B}^{1}\left(\mathbf{R}^{d}\right), i=\overline{1, d}, j=\overline{1, r}$. Tozda

$$
\sup _{t \in T} \mathbf{E}\left\|Y_{n}(t, \omega)-X(t, \omega)\right\|^{2} \leqslant C \sup _{t \in\left[0, h_{n}\right)} \mathbf{E}\left\|x-X_{0 n}(t, \omega)\right\|^{2}+C h_{n}+\frac{C}{n h_{n}^{2}},
$$

где $X(t, \omega), Y_{n}(t, \omega), t \in T, \omega \in \Omega, n \in \mathbf{N},-$ решения задач (1.1) $u(1.16)$ соответственно, $1 / n<h_{n}^{2}$.

Д о к а з а т е л ь с т в о. Решение (1.16) запишем в виде

$$
\begin{aligned}
Y_{n}^{i}(\tau, \omega)= & X_{0 n}^{i}\left(t_{\tau}, \omega\right)+\sum_{j=1}^{r} \sum_{l=1}^{k_{\tau}} \sigma_{n}^{i j}\left(Y_{n}\left(t_{\tau}+(l-1) h_{n}, \omega\right)\right) \\
& \times\left[B_{n}^{j}\left(t_{\tau}+l h_{n}, \omega\right)-B_{n}^{j}\left(t_{\tau}+(l-1) h_{n}, \omega\right)\right] \\
+ & \sum_{l=1}^{k_{\tau}} a_{n}^{i}\left(Y_{n}\left(t_{\tau}+(l-1) h_{n}, \omega\right)\right) h_{n}, \quad i=\overline{1, d}
\end{aligned}
$$


Пусть $X(t, \omega)$ и $X_{n}(t, \omega)$ - решения задач (1.1) и (1.3) соответственно, тогда

$$
\begin{aligned}
& \left\|X(\tau, \omega)-Y_{n}(\tau, \omega)\right\| \leqslant\left\|X(\tau, \omega)-X_{n}(\tau, \omega)\right\| \\
& +\left\|X_{n}(\tau, \omega)-Y_{n}(\tau, \omega)\right\| \\
& \left\|X_{n}(\tau, \omega)-Y_{n}(\tau, \omega)\right\| \\
& \leqslant \| \sum_{j=1}^{r} \sum_{l=1}^{k_{\tau}}\left[\sigma_{n}^{j}\left(X_{n}\left(t_{\tau}+(l-2) h_{n}, \omega\right)\right)-\sigma_{n}^{j}\left(Y_{n}\left(t_{\tau}+(l-1) h_{n}, \omega\right)\right)\right] \\
& \times\left[B_{n}^{i}\left(t_{\tau}+l h_{n}, \omega\right)-B_{n}^{j}\left(t_{\tau}+(l-1) h_{n}, \omega\right)\right] \| \\
& +\left\|\sum_{l=1}^{k_{\tau}}\left[a_{n}\left(X_{n}\left(t_{\tau}+(l-1) h_{n}, \omega\right)\right)-a_{n}\left(Y_{n}\left(t_{\tau}+(l-1) h_{n}, \omega\right)\right)\right] h_{n}\right\| \\
& =J_{1}(\tau, \omega)+J_{2}(\tau, \omega) \text {. }
\end{aligned}
$$

Для оценки $J_{1}(\tau, \omega)$ используем методику оценивания неравенства (1.5) и мартингальное неравенство [13, с. 41]:

$\mathbf{E}\left[J_{1}(\tau, \omega)\right]^{2} \leqslant \frac{C k_{a}}{n}+\frac{C k_{a}^{2}}{n}+C h_{n}$

$$
+C h_{n} \sum_{l=2}^{k_{\tau}} \mathbf{E}\left\|X_{n}\left(t_{\tau}+(l-2) h_{n}, \omega\right)-Y_{n}\left(t_{\tau}+(l-2) h_{n}, \omega\right)\right\|^{2} \text {. }
$$

Для $J_{2}(\tau, \omega)$ получим

$$
\begin{aligned}
\mathbf{E}\left[J_{2}(\tau, \omega)\right]^{2} \leqslant C h_{n} \sum_{l=1}^{k_{\tau}} \mathbf{E} \| & X_{n}\left(t_{\tau}+(l-1) h_{n}, \omega\right) \\
& -Y_{n}\left(t_{\tau}+(l-1) h_{n}, \omega\right) \|^{2},
\end{aligned}
$$

а из (1.18)-(1.20) вытекает

$$
\begin{aligned}
& \mathbf{E}\left\|X_{n}(\tau, \omega)-Y_{n}(\tau, \omega)\right\|^{2} \\
& \leqslant C h_{n} \sum_{l=1}^{k_{\tau}} \mathbf{E}\left\|X_{n}\left(t_{\tau}+(l-1) h_{n}, \omega\right)-Y_{n}\left(t_{\tau}+(l-1) h_{n}, \omega\right)\right\|^{2} \\
& \quad+\frac{C k_{a}^{2}}{n}+C h_{n} .
\end{aligned}
$$

$\mathrm{K}$ неравенству (1.21) применим утверждение (1.14). Тогда

$$
\mathbf{E}\left\|X_{n}(\tau, \omega)-Y_{n}(\tau, \omega)\right\|^{2} \leqslant \frac{C k_{a}^{2}}{n}+C h_{n} .
$$

Из (1.17), (1.22) и теоремы 1 следует утверждение теоремы 2. 
Аналогично доказывается

Теорема 3. При выполнении условий теоремь 2 имеет место следующее неравенство:

$\sup _{t \in T} \mathbf{E}\left\|Y_{n}(t, \omega)-X(t, \omega)\right\|^{2} \leqslant C \sup _{t \in\left[0, h_{n}\right)} \mathbf{E}\left\|x-X_{0 n}(t, \omega)\right\|^{2}+C h_{n}^{1 / 5}+\frac{C}{n h_{n}}$, где $X(t, \omega), Y_{n}(t, \omega), t \in T, \omega \in \Omega, n \in \mathbf{N}$, - решения уравнений (1.1) $u$ (1.16) соответственно, $1 / n<h_{n}$.

3 а м е ч а н и е 3. Сравним результаты теорем 2 и 3 . Несложно видеть, что в теореме $2 Y_{n}(t, \omega)$ сходится к $X(t, \omega)$ равномерно по $t \in T$, если $1 / n=o\left(h_{n}^{2}\right), n \rightarrow \infty, h_{n} \rightarrow 0$, а в теореме 3 подобный результат достигается, если $1 / n=o\left(h_{n}\right)$ (см. следствие 2). Таким образом, теорема 3 расширяет «область» Ито до оптимального размера. С другой стороны, в правой части неравенства из теоремы 3 стоит слагаемое $C h_{n}^{1 / 5}$, а в теореме 2 аналогичное слагаемое имеет вид $C h_{n}$, т.е. при соответствуюшем выборе связи между $1 / n$ и $h_{n}$ (например, $1 / n \sim h_{n}^{3}$ ) оценка в теореме 2 будет лучше, нежели та, которую дает теорема 3.

Из теоремы 3 вытекает

Следствие 2. Пуусть $\sigma^{i j} \in C_{B}^{2}\left(\mathbf{R}^{d}\right), a^{i} \in C_{B}^{1}\left(\mathbf{R}^{d}\right), i=\overline{1, d}, j=\overline{1, r}$, $u n \rightarrow \infty, h_{n} \rightarrow 0$ max, umo

$$
\begin{gathered}
n^{-1}=o\left(h_{n}\right), \\
\sup _{t \in\left[0, h_{n}\right)} \mathbf{E}\left\|x-X_{0 n}(t, \omega)\right\|^{2} \longrightarrow 0 .
\end{gathered}
$$

$T o z \partial a \sup _{t \in T} \mathrm{E}\left\|Y_{n}(t, \omega)-X(t, \omega)\right\|^{2} \longrightarrow 0$, , дде $X(t, \omega) u Y_{n}(t, \omega), t \in T$, $\omega \in \Omega, n \in \mathbf{N}$, - решения задач (1.1) и (1.16) соответственно.

1.3. Аппроксимация решений стохастических дифференциальных уравнений Стратоновича решениями конечно-разностных уравнений с осреднением. Рассмотрим систему стохастических дифференциальных уравнений

$$
\begin{aligned}
X^{i}(t, \omega)=x^{i} & +\sum_{j=1}^{r}(S) \int_{0}^{t} \sigma^{i j}(X(s, \omega)) d \dot{B^{j}}(s, \omega) \\
& +\int_{0}^{t} a^{i}(X(s, \omega)) d s, \quad i=\overline{1, d}
\end{aligned}
$$

где $x, \sigma^{i j}, a^{i}, i=\overline{1, d}, j=\overline{1, r}$, задаются так же, как и в системе (1.1), а интеграл в правой части (1.24) - стохастический интеграл Стратоновича $[13$, с. 109].

Существование и единственность решения (1.24) доказаны в $[13$, c. 233].

Пусть $B_{n}^{j}(t, \omega), j=\overline{1, r}$, - выражение из соотношений (1.2). Тогда справедлива 
Лемма ([11]). Для достаточно больших $n u h_{n}<1 / n$ выполндются следующие оченки $(k \in \mathbf{N}, j=\overline{1, r})$ :
a) $\mathbf{E}\left[B_{n}^{j}\left(k h_{n}, \omega\right)-B_{n}^{j}\left((k-1) h_{n}, \omega\right)\right]^{2} \leqslant C n h_{n}^{2}$,
б) $\mathbf{E}\left[B_{n}^{j}\left(k h_{n}, \omega\right)-B_{n}^{j}\left((k-1) h_{n}, \omega\right)\right]^{4} \leqslant C n^{2} h_{n}^{4}$,
в) $\mathbf{E}\left[B_{n}^{j}\left(k h_{n}, \omega\right)-B_{n}^{j}\left((k-1) h_{n}, \omega\right)\right]^{8} \leqslant C n^{4} h_{n}^{8}$. Тогда

Теорема 4. Пусть $\sigma^{i j} \in C_{B}^{2}\left(\mathbf{R}^{d}\right), a^{i} \in C_{B}^{1}\left(\mathbf{R}^{d}\right), i=\overline{1, d}, j=\overline{1, r}$. $\sup _{t \in T} \mathbf{E}\left\|X(t, \omega)-Y_{n}(t, \omega)\right\|^{2} \leqslant C n^{-1 / 5}+C n^{2} h_{n}^{2}+C \sup _{t \in\left[0, h_{n}\right)} \mathbf{E}\left\|X_{0 n}(t, \omega)-x\right\|^{2}$, где $X(t, \omega) u Y_{n}(t, \omega)$ - решения задач (1.24) и (1.16) соответственно, $h_{n}<1 / n$.

Д о к а $а$ а те ль с т в о. Пусть по определению $\widetilde{\delta}=l h_{n}, l \in \mathbf{N}$, и $1 / n=o(\widetilde{\delta})$ при $n \rightarrow \infty$. Тогда для любого $\tau \in T$ найдутся такие $t_{\tau} \in\left[0, h_{n}\right)$ и $t_{\tau}^{\prime} \in[\widetilde{\delta}, 2 \widetilde{\delta})$, а также $k_{\tau} \in \mathbf{Z}_{+}$и $m_{\tau} \in \mathbf{N}$, что, с одной стороны, $\tau=t_{\tau}+k_{\tau} h_{n}$, а с другой, $\tau=t_{\tau}^{\prime}+m_{\tau} \tilde{\delta}$, где $t_{\tau}^{\prime}=t_{\tau}+k_{\tau}^{\prime} h_{n}$ и $k_{\tau}^{\prime} \in \mathbf{N}, k_{\tau}^{\prime}<2 l$.

Из уравнения (1.16) методом конечных шагов получаем

$$
\begin{aligned}
Y_{n}^{i}(\tau, \omega)= & \left\{X_{0 n}^{i}\left(t_{\tau}, \omega\right)+\sum_{j=1}^{r} \sum_{k=1}^{k_{\tau}^{\prime}} \sigma_{n}^{i j}\left(Y_{n}\left(t_{\tau}+(k-1) h_{n}, \omega\right)\right)\right. \\
& \times\left[B_{n}^{j}\left(t_{\tau}+k h_{n}, \omega\right)-B_{n}^{j}\left(t_{\tau}+(k-1) h_{n}, \omega\right)\right] \\
& \left.+\sum_{k=1}^{k_{\tau}^{\prime}} a_{n}^{i}\left(Y_{n}\left(t_{\tau}+(k-1) h_{n}, \omega\right)\right) h_{n}\right\} \\
+ & \left\{\sum_{j=1}^{r} \sum_{k=0}^{m_{\tau}-1} \sum_{p=1}^{l} \sigma_{n}^{i j}\left(Y_{n}\left(t_{\tau}^{\prime}+k \widetilde{\delta}+(p-1) h_{n}, \omega\right)\right)\right. \\
& \times\left[B_{n}^{j}\left(t_{\tau}^{\prime}+k \widetilde{\delta}+p h_{n}, \omega\right)-B_{n}^{j}\left(t_{\tau}^{\prime}+k \widetilde{\delta}+(p-1) h_{n}, \omega\right)\right] \\
& \left.\quad+\sum_{k=0}^{m_{\tau}-1} \sum_{p=1}^{l} a_{n}^{i}\left(Y_{n}\left(t_{\tau}^{\prime}+k \widetilde{\delta}+(p-1) h_{n}, \omega\right)\right) h_{n}\right\} \\
= & S_{1}^{i}(\tau, \omega)+S_{2}^{i}(\tau, \omega) .
\end{aligned}
$$

Из монографии $[13$, с. 394$]$ известно, что интегралы Ито и Стратоновича связаны соотношением

$$
\begin{aligned}
& (S) \int_{0}^{\tau} \sigma^{i j}(X(s, \omega)) d B^{j}(s, \omega)=(I) \int_{0}^{\tau} \sigma^{i j}(X(s, \omega)) d B^{j}(s, \omega) \\
& +\frac{1}{2} \sum_{\alpha=1}^{d} \int_{0}^{\tau}\left(\sigma^{\alpha j} \partial_{\alpha} \sigma^{i j}\right)(X(s, \omega)) d s
\end{aligned}
$$


Используя соотношения (1.25), (1.26), введем следуюшие обозначения: для $i=1, \ldots, d$

$$
\begin{aligned}
& H_{1}^{i}(\tau, \omega)=Y_{n}^{i}\left(t_{\tau}^{\prime}, \omega\right)-X^{i}\left(t_{\tau}^{\prime}, \omega\right) \\
& =S_{1}^{i}(\tau, \omega)-x^{i}-\sum_{j=1}^{r}(I) \int_{0}^{t_{\tau}^{\prime}} \sigma^{i j}(X(s, \omega)) d B^{j}(s, \omega) \\
& \quad-\frac{1}{2} \sum_{j=1}^{r} \sum_{\alpha=1}^{d} \int_{0}^{t_{\tau}^{\prime}}\left(\sigma^{\alpha j} \partial_{\alpha} \sigma^{i j}\right)(X(s, \omega)) d s-\int_{0}^{t_{\tau}^{\prime}} a^{i}(X(s, \omega)) d s \\
& H_{2}^{i}(\tau, \omega)=S_{2}^{i}(\tau, \omega)-\sum_{j=1}^{r}(I) \int_{t_{\tau}^{\prime}}^{\tau} \sigma^{i j}(X(s, \omega)) d B^{j}(s, \omega) \\
& \quad-\frac{1}{2} \sum_{j=1}^{r} \sum_{\alpha=1}^{d} \int_{t_{\tau}^{\prime}}^{\tau}\left(\sigma^{\alpha j} \partial_{\alpha} \sigma^{i j}\right)(X(s, \omega)) d s-\int_{t_{\tau}^{\prime}}^{\tau} a^{i}(X(s, \omega)) d s
\end{aligned}
$$

Начнем наши исследования с $H_{2}^{i}(\tau, \omega)$, где $i$ произвольное, но фиксированное натуральное число. Очевидно следующее равенство:

$$
H_{2}^{i}(\tau, \omega)=I_{0}(\tau, \omega)+I_{1}(\tau, \omega)+I_{2}(\tau, \omega)+I_{3}(\tau, \omega)+I_{4}(\tau, \omega),
$$

где

$$
\begin{aligned}
& I_{0}(\tau, \omega)=\sum_{j=1}^{\tau} \sum_{k=0}^{m_{\tau}-1} \sum_{p=1}^{l}\left[\sigma_{n}^{i j}\left(Y_{n}\left(t_{\tau}^{\prime}+k \tilde{\delta}+(p-1) h_{n}, \omega\right)\right)\right. \\
& \left.-\sigma^{i j}\left(Y_{n}\left(t_{\tau}^{\prime}+k \tilde{\delta}+(p-1) h_{n}, \omega\right)\right)\right] \\
& \times\left[B_{n}^{j}\left(t_{\tau}^{\prime}+k \widetilde{\delta}+p h_{n}, \omega\right)-B_{n}^{j}\left(t_{\tau}^{\prime}+k \widetilde{\delta}+(p-1) h_{n}, \omega\right)\right], \\
& I_{1}(\tau, \omega)=\sum_{j=1}^{r} \sum_{k=0}^{m_{\tau}-1} \sigma^{i j}\left(Y_{n}\left(t_{\tau}^{\prime}+k \widetilde{\delta}-\delta_{1}, \omega\right)\right) \\
& \times\left[B^{j}\left(t_{\tau}^{\prime}+(k+1) \tilde{\delta}, \omega\right)-B^{j}\left(t_{\tau}^{\prime}+k \widetilde{\delta}, \omega\right)\right] \\
& -\sum_{j=1}^{r}(I) \int_{t_{\tau}^{\prime}}^{\tau} \sigma^{i j}(X(s, \omega)) d B^{j}(s, \omega) \\
& \delta_{1}=\lambda h_{n}, \quad \lambda=\left[\frac{1 / n+h_{n}}{h_{n}}\right] \in \mathbf{N}, \\
& I_{2}(\tau, \omega)=\sum_{j=1}^{r} \sum_{k=0}^{m_{\tau}-1} \sum_{p=1}^{l}\left[\sigma^{i j}\left(Y_{n}\left(t_{\tau}^{\prime}+k \tilde{\delta}+(p-1) h_{n}, \omega\right)\right)\right. \\
& \left.-\sigma^{i j}\left(Y_{n}\left(t_{\tau}^{\prime}+k \widetilde{\delta}, \omega\right)\right)\right] \\
& \times\left[B_{n}^{j}\left(t_{\tau}^{\prime}+k \widetilde{\delta}+p h_{n}, \omega\right)-B_{n}^{j}\left(t_{\tau}^{\prime}+k \widetilde{\delta}+(p-1) h_{n}, \omega\right)\right]
\end{aligned}
$$




$$
\begin{aligned}
& -\frac{1}{2} \sum_{j=1}^{r} \sum_{\alpha=1}^{d} \int_{t_{\tau}^{\prime}}^{\tau}\left(\sigma^{\alpha j} \partial_{\alpha} \sigma^{i j}\right)(X(s, \omega)) d s \\
I_{3}(\tau, \omega)= & \sum_{j=1}^{r} \sum_{k=0}^{m_{\tau}-1} \sigma^{i j}\left(Y_{n}\left(t_{\tau}^{\prime}+k \tilde{\delta}, \omega\right)\right) \\
& \times\left[B_{n}^{j}\left(t_{\tau}^{\prime}+(k+1) \widetilde{\delta}, \omega\right)-B_{n}^{j}\left(t_{\tau}^{\prime}+k \widetilde{\delta}, \omega\right)\right] \\
& -\sum_{j=1}^{r} \sum_{k=0}^{m_{\tau}-1} \sigma^{i j}\left(Y_{n}\left(t_{\tau}^{\prime}+k \widetilde{\delta}-\delta_{1}, \omega\right)\right) \\
I_{4}(\tau, \omega)= & \sum_{k=0}^{m_{\tau}-1} \sum_{p=1}^{l} a_{n}^{i}\left(Y_{n}\left(t_{\tau}^{\prime}+k \tilde{\delta}+(p-1) h_{n}, \omega\right)\right) h_{n} \\
& -\int_{t_{\tau}^{\prime}}^{\tau} a^{i}(X(s, \omega)) d s .
\end{aligned}
$$

Оценим $I_{0}(\tau, \omega)$. Воспользовавшись неравенством Гёльдерӓ, результатами леммы и представлением $\sigma_{n}^{i j}$, получим

$$
\mathbf{E}\left\{I_{0}(\tau, \omega)\right\}^{2} \leqslant C n^{-1}
$$

Прх оценивании $I_{3}(\tau, \omega)$ учитываем независимость сомножителей под знаком суммы и ограниченность функции $\sigma^{i j}, i=\overline{1, d}, j=\overline{1, r}$. В результате имеем

$$
\mathrm{E}\left\{I_{3}(\tau, \omega)\right\}^{2} \leqslant C(n \widetilde{\delta})^{-1}
$$

Рассмотрим $I_{4}(\tau, \omega)$. При оценивании воспользуемся видом $a_{n}^{i}$, неравенством Гёльдера, многомерной формулой Лагранжа и тем, что первая сумма в представлении $Y_{n}^{\alpha}, \alpha=\overline{1, d}$, мажорирует вторую:

$$
\begin{aligned}
\mathbf{E}\left\{I_{4}(\tau, \omega)\right\}^{2} \leqslant & \frac{C}{n^{2}}+C \widetilde{\delta}+C n \tilde{\delta}^{2}+C \tilde{\delta} \\
& \times \sum_{\alpha=1}^{d} \sum_{k=0}^{m_{\tau}-1} \mathbf{E}\left|Y_{n}^{\alpha}\left(t_{\tau}^{\prime}+k \tilde{\delta}, \omega\right)-X^{\alpha}\left(t_{\tau}^{\prime}+k \tilde{\delta}, \omega\right)\right|^{2}
\end{aligned}
$$

Перейдем к исследованию $I_{2}(\tau, \omega)$. Прежде всего, перепишем исходное выражение следующим образом:

$$
\begin{gathered}
I_{2}(\tau, \omega)=\left\{\sum _ { j = 1 } ^ { r } \sum _ { k = 0 } ^ { m _ { \tau } - 1 } \sum _ { p = 1 } ^ { l } \left[\sigma^{i j}\left(Y_{n}\left(t_{\tau}^{\prime}+k \widetilde{\delta}+(p-1) h_{n}, \omega\right)\right)\right.\right. \\
\left.-\sigma^{i j}\left(Y_{n}\left(t_{\tau}^{\prime}+k \widetilde{\delta}, \omega\right)\right)\right]
\end{gathered}
$$




$$
\begin{aligned}
& \times {\left[B_{n}^{j}\left(t_{\tau}^{\prime}+k \widetilde{\delta}+p h_{n}, \omega\right)-B_{n}^{j}\left(t_{\tau}^{\prime}+k \widetilde{\delta}+(p-1) h_{n}, \omega\right)\right] } \\
&-\frac{1}{2} \sum_{j=1}^{r} \sum_{k=0}^{m_{\tau}-1} \sum_{\alpha=1}^{d}\left(\sigma^{\alpha j} \partial_{\alpha} \sigma^{i j}\right)\left(Y_{n}\left(t_{\tau}^{\prime}+k \widetilde{\delta}, \omega\right)\right) \\
&\left.\times\left[B_{n}^{j}\left(t_{\tau}^{\prime}+(k+1) \tilde{\delta}, \omega\right)-B_{n}^{j}\left(t_{\tau}^{\prime}+k \widetilde{\delta}, \omega\right)\right]^{2}\right\} \\
&+ \frac{1}{2} \sum_{j=1}^{r} \sum_{k=0}^{m_{\tau}-1} \sum_{\alpha=1}^{d}\left(\sigma^{\alpha j} \partial_{\alpha} \sigma^{i j}\right)\left(Y_{n}\left(t_{\tau}^{\prime}+k \widetilde{\delta}, \omega\right)\right) \\
& \times\left[B_{n}^{j}\left(t_{\tau}^{\prime}+(k+1) \tilde{\delta}, \omega\right)-B_{n}^{j}\left(t_{\tau}^{\prime}+k \widetilde{\delta}, \omega\right)\right]^{2} \\
&\left.-\frac{1}{2} \sum_{j=1}^{r} \sum_{\alpha=1}^{d} \int_{t_{\tau}^{\prime}}^{\tau}\left(\sigma^{\alpha j} \partial_{\alpha} \sigma^{i j}\right)(X(s, \omega)) d s\right\} \\
&=I_{21}(\tau, \omega)+\frac{1}{2} I_{22}(\tau, \omega) .
\end{aligned}
$$

Пользуясь неравенством Гёльдера, результатами леммы, представлением $X^{\alpha}(s, \omega), \alpha=\overline{1, d}$, многомерной формулой Лагранжа, свойствами интеграла Ито и тем, что $\sigma^{i j} \in C_{B}^{2}\left(\mathbf{R}^{d}\right), i=\overline{1, d}, j=\overline{1, r}$, можно доказать справедливость неравенства

$$
\begin{aligned}
\mathbf{E}\left\{I_{22}(\tau, \omega)\right\}^{2} \leqslant & \frac{C}{n \widetilde{\delta}}+C \Delta+\frac{C}{n} \\
& +C \widetilde{\delta} \sum_{k=0}^{m_{\tau}-1} \sum_{\alpha=1}^{d} \mathbf{E}\left|Y_{n}^{\alpha}\left(t_{\tau}^{\prime}+k \widetilde{\delta}, \omega\right)-X^{\alpha}\left(t_{\tau}^{\prime}+k \widetilde{\delta}\right)\right|^{2}
\end{aligned}
$$

где $\Delta=b \widetilde{\delta}, b \in \mathbf{N}$ и $\tau=t_{\tau}^{\prime}+a_{\tau} \Delta$.

Приступим к оценке $I_{21}(\tau, \omega)$. Выпишем очевидную оценку сверху для этого выражения в $L^{2}(\Omega, \mathscr{A}, \mathbf{P})$ :

$$
\mathbf{E}\left\{I_{21}(\tau, \omega)\right\}^{2} \leqslant I_{21}^{1}(\tau)+I_{21}^{2}(\tau)
$$

где

$$
\begin{aligned}
I_{21}^{1}(\tau)=C \mathbf{E} & \left\{\sum _ { j = 1 } ^ { r } \sum _ { k = 0 } ^ { m _ { \tau } - 1 } \sum _ { p = 1 } ^ { l } \left[\sigma^{i j}\left(Y_{n}\left(t_{\tau}^{\prime}+k \widetilde{\delta}+(p-1) h_{n}, \omega\right)\right)\right.\right. \\
& \left.-\sigma^{i j}\left(Y_{n}\left(t_{\tau}^{\prime}+k \tilde{\delta}, \omega\right)\right)\right] \\
& \times\left[B_{n}^{j}\left(t_{\tau}^{\prime}+k \widetilde{\delta}+p h_{n}, \omega\right)-B_{n}^{j}\left(t_{\tau}^{\prime}+k \widetilde{\delta}+(p-1) h_{n}, \omega\right)\right] \\
& -\sum_{j=1}^{r} \sum_{k=0}^{m_{\tau}-1} \sum_{p=1}^{l} \sum_{\alpha=1}^{d}\left(\sigma^{\alpha j} \partial_{\alpha} \sigma^{i j}\right)\left(Y_{n}\left(t_{\tau}^{\prime}+k \widetilde{\delta}, \omega\right)\right)
\end{aligned}
$$




$$
\begin{aligned}
& \times\left[B_{n}^{j}\left(t_{\tau}^{\prime}+k \widetilde{\delta}+(p-1) h_{n}, \omega\right)-B_{n}^{j}\left(t_{\tau}^{\prime}+k \widetilde{\delta}, \omega\right)\right] \\
& \left.\times\left[B_{n}^{j}\left(t_{\tau}^{\prime}+k \widetilde{\delta}+p h_{n}, \omega\right)-B_{n}^{j}\left(t_{\tau}^{\prime}+k \widetilde{\delta}+(p-1) h_{n}, \omega\right)\right]\right\}^{2}, \\
I_{21}^{2}(\tau)=C \mathbf{E} & \left\{\sum_{j=1}^{r} \sum_{k=0}^{m_{\tau}-1} \sum_{p=1}^{l} \sum_{\alpha=1}^{d}\left(\sigma^{\alpha j} \partial_{\alpha} \sigma^{i j}\right)\left(Y_{n}\left(t_{\tau}^{\prime}+k \widetilde{\delta}, \omega\right)\right)\right. \\
& \times\left[B_{n}^{j}\left(t_{\tau}^{\prime}+k \widetilde{\delta}+(p-1) h_{n}, \omega\right)-B_{n}^{j}\left(t_{\tau}^{\prime}+k \widetilde{\delta}, \omega\right)\right] \\
& \times\left[B_{n}^{j}\left(t_{\tau}^{\prime}+k \widetilde{\delta}+p h_{n}, \omega\right)-B_{n}^{j}\left(t_{\tau}^{\prime}+k \widetilde{\delta}+(p-1) h_{n}, \omega\right)\right] \\
& -\frac{1}{2} \sum_{j=1}^{r} \sum_{k=0}^{m_{\tau}-1} \sum_{\alpha=1}^{d}\left(\sigma^{\alpha j} \partial_{\alpha} \sigma^{i j}\right)\left(Y_{n}\left(t_{\tau}^{\prime}+k \widetilde{\delta}, \psi\right)\right) \\
& \left.\times\left[B_{n}^{j}\left(t_{\tau}^{\prime}+(k+1) \widetilde{\delta}, \omega\right)-B_{n}^{j}\left(t_{\tau}^{\prime}+k \widetilde{\delta}, \omega\right)\right]^{2}\right\}^{2}
\end{aligned}
$$

Исследуем $I_{21}^{1}(\tau)$. Используя ту же методику, что и при доказательстве неравенства (1.34), получим

$$
I_{21}^{1}(\tau) \leqslant C n^{3} \widetilde{\delta}^{4}+C n \widetilde{\delta}^{2}+C \widetilde{\delta}
$$

Оценим $I_{21}^{2}(\tau)$. Прежде всего выпишем тождество, которое мы будем использовать в дальнейшем:

$$
\begin{aligned}
\sum_{p=1}^{l} & {\left[B_{n}^{j}\left(t_{\tau}^{\prime}+k \widetilde{\delta}+(p-1) h_{n}, \omega\right)-B_{n}^{j}\left(t_{\tau}^{\prime}+k \widetilde{\delta}, \omega\right)\right] } \\
& \times\left[B_{n}^{j}\left(t_{\tau}^{\prime}+k \widetilde{\delta}+p h_{n}, \omega\right)-B_{n}^{j}\left(t_{\tau}^{\prime}+k \widetilde{\delta}+(p-1) h_{n}, \omega\right)\right] \\
= & \frac{1}{2}\left[B_{n}^{j}\left(t_{\tau}^{\prime}+(k+1) \widetilde{\delta}, \omega\right)-B_{n}^{j}\left(t_{\tau}^{\prime}+k \widetilde{\delta}, \omega\right)\right]^{2} \\
& -\frac{1}{2} \sum_{p=1}^{l}\left[B_{n}^{j}\left(t_{\tau}^{\prime}+k \widetilde{\delta}+p h_{n}, \omega\right)-B_{n}^{j}\left(t_{\tau}^{\prime}+k \widetilde{\delta}+(p-1) h_{n}, \omega\right)\right]^{2}
\end{aligned}
$$

Из данного тождества и леммы вытекает, что

$$
I_{21}^{2}(\tau) \leqslant C n^{2} h_{n}^{2}
$$

Из соотношений (1.35)-(1.37) следует

$$
\mathbf{E}\left\{I_{21}(\tau, \omega)\right\}^{2} \leqslant C n^{3} \widetilde{\delta}^{4}+C n \widetilde{\delta}^{2}+C \widetilde{\delta}+C n^{2} h_{n}^{2}
$$


а из соотношений $(1.33),(1.34),(1.38)$ получаем

$$
\begin{aligned}
\mathbf{E}\left\{I_{2}(\tau, \omega)\right\}^{2} \leqslant & C n^{3} \widetilde{\delta}^{4}+C n \widetilde{\delta}^{2} \\
& +C \dot{\tilde{\delta}^{2}} \sum_{k=0}^{m_{\tau}-1} \sum_{\alpha=1}^{d} \mathbf{E}\left|Y_{n}^{\alpha}\left(t_{\tau}^{\prime}+k \widetilde{\delta}, \omega\right)-X^{\alpha}\left(t_{\tau}^{\prime}+k \widetilde{\delta}, \omega\right)\right|^{2} \\
& +C n^{2} h_{n}^{2}+\frac{C}{n \widetilde{\delta}}+C \Delta+\frac{C}{n}
\end{aligned}
$$

Исследуем $I_{1}(\tau, \omega)$. Очевидно следующее представление:

$$
\begin{aligned}
I_{1}(\tau, \omega)= & \sum_{j=1}^{r} \sum_{k=0}^{m_{\tau}-1}\left[\sigma^{i j}\left(Y_{n}\left(t_{\tau}^{\prime}+k \widetilde{\delta}-\delta_{1}, \omega\right)\right)-\sigma^{i j}\left(X\left(t_{\tau}^{\prime}+k \widetilde{\delta}, \omega\right)\right)\right] \\
& \times\left[B^{j}\left(t_{\tau}^{\prime}+(k+1) \widetilde{\delta}, \omega\right)-B^{j}\left(t_{\tau}^{\prime}+k \widetilde{\delta}, \omega\right)\right] \\
+ & \left\{\sum_{j=1}^{r} \sum_{k=0}^{m_{\tau}-1} \sigma^{i j}\left(X\left(t_{\tau}^{\prime}+k \widetilde{\delta}, \omega\right)\right)\right. \\
& \times\left[B^{j}\left(t_{\tau}^{\prime}+(k+1) \tilde{\delta}, \omega\right)-B^{j}\left(t_{\tau}^{\prime}+k \widetilde{\delta}, \omega\right)\right] \\
& \left.-\sum_{j=1}^{r}(I) \int_{t_{\tau}^{\prime}}^{\tau} \sigma^{i j}(X(s, \omega)) d B^{j}(s, \omega)\right\} \\
= & I_{11}(\tau, \omega)+I_{12}(\tau, \omega)
\end{aligned}
$$

Оценим $I_{11}(\tau, \omega)$ в $L^{2}(\Omega, \mathscr{A}, \mathbf{P})$. Для этого воспользуемся независимостью сомножителей в этом выражении, видом $Y_{n}^{\alpha}, \alpha=\overline{1, d}$, и формулой Лагранжа. В результате получим

$$
\begin{array}{r}
\mathbf{E}\left\{I_{11}(\tau, \omega)\right\}^{2} \leqslant \frac{C}{n}+C \widetilde{\delta} \sum_{k=0}^{m_{\tau}-1} \sum_{\alpha=1}^{d} \mathbf{E} \mid Y_{n}^{\alpha}\left(t_{\tau}^{\prime}+k \widetilde{\delta}, \omega\right) \\
-\left.X^{\alpha}\left(t_{\tau}^{\prime}+k \widetilde{\delta}, \omega\right)\right|^{2}
\end{array}
$$

При оценивании $I_{12}(\tau, \omega)$ будем пользоваться определением интеграла Ито и видом $X(\tau, \omega)$, тогда

$$
\begin{gathered}
\mathbf{E}\left\{I_{12}(\tau, \omega)\right\}^{2}=\mathbf{E}\left\{\sum _ { j = 1 } ^ { r } \sum _ { k = 0 } ^ { m _ { \tau } - 1 } ( I ) \int _ { t _ { \tau } ^ { \prime } + k \tilde { \delta } } ^ { t _ { \tau } ^ { \prime } + ( k + 1 ) \widetilde { \delta } } \left[\sigma^{i j}\left(X\left(t_{\tau}^{\prime}+k \tilde{\delta}, \omega\right)\right)\right.\right. \\
\left.\left.-\sigma^{i j}(X(s, \omega))\right] d B^{j}(s, \omega)\right\}^{2} \leqslant C \widetilde{\delta}
\end{gathered}
$$


Из соотношений (1.40)-(1.42) следует, что

$$
\begin{aligned}
& \mathbf{E}\left\{I_{1}(\tau, \omega)\right\}^{2} \leqslant \frac{C}{n}+C \tilde{\delta} \\
& \quad+C \tilde{\delta} \sum_{k=0}^{m_{\tau}-1} \sum_{\alpha=1}^{d} \mathbf{E}\left|Y_{n}^{\alpha}\left(t_{\tau}^{\prime}+k \tilde{\delta}, \omega\right)-X^{\alpha}\left(t_{\tau}^{\prime}+k \widetilde{\delta}, \omega\right)\right|^{2}
\end{aligned}
$$

а из соотношений $(1.28)-(1.32),(1.39),(1.43)$ получаем для $i=\overline{1, d}$

$$
\begin{array}{r}
\mathbf{E}\left\{H_{2}^{i}(\tau, \omega)\right\}^{2} \leqslant \frac{C}{n}+\frac{C}{n \widetilde{\delta}}+C n \widetilde{\delta}^{2}+C n^{3} \widetilde{\delta}^{4}+C n^{2} h_{n}^{4}+C \Delta \\
+C \widetilde{\delta} \sum_{k=0}^{m_{\tau}-1} \sum_{\alpha=1}^{d} \mathbf{E}\left|Y_{n}^{\alpha}\left(t_{\tau}^{\prime}+k \widetilde{\delta}, \omega\right)-X^{\alpha}\left(t_{\tau}^{\prime}+k \widetilde{\delta}, \omega\right)\right|^{2} .
\end{array}
$$

Далее воспользуемся утверждением: если $x_{k} \leqslant a+b \sum_{j=0}^{k-1} x_{j}$, то $x_{k} \leqslant(b+1)^{k-1}\left(a+b x_{0}\right)$.

Применив это утверждение к неравенству (1.44), получим

$$
\begin{aligned}
& \mathbf{E}\left\|Y_{n}\left(t_{\tau}^{\prime}+m_{\tau} \widetilde{\delta}, \omega\right)-X\left(t_{\tau}^{\prime}+m_{\tau} \widetilde{\delta}, \omega\right)\right\|^{2} \\
& \leqslant C \sum_{i=1}^{d} \mathbf{E}\left\{H_{1}^{i}(\tau, \omega)\right\}^{2}+\frac{C}{n}+\frac{C}{n \widetilde{\delta}}+C n \widetilde{\delta}^{2}+C n^{3} \widetilde{\delta}^{4}+C n^{2} h_{n}^{2}+C \Delta \\
& \quad+C \widetilde{\delta} \sum_{i=1}^{d} \mathbf{E}\left|Y_{n}^{i}\left(t_{\tau}^{\prime}, \omega\right)-X^{i}\left(t_{\tau}^{\prime}, \omega\right)\right|^{2} .
\end{aligned}
$$

Оценим $H_{1}^{i}(\tau, \omega)$ из (1.27). С учетом леммы получаем, что

$\mathbf{E}\left\{H_{1}^{i}(\tau, \omega)\right\}^{2} \leqslant C \sup _{t \in\left[0, h_{n}\right)} \mathbf{E}\left|X_{0 n}^{i}(t, \omega)-x^{i}\right|^{2}+C n \tilde{\delta}^{2}+C \tilde{\delta}$.

Из неравенств (1.45), (1.46) вытекает, что

$$
\begin{aligned}
\sup _{t \in T} \mathbf{E}\left\|Y_{n}(t, \omega)-X(t, \omega)\right\|^{2} \leqslant & \frac{C}{n}+\frac{C}{n \widetilde{\delta}}+C n \widetilde{\delta}^{2}+C n^{3} \widetilde{\delta}^{4}+C n^{2} h_{n}^{2}+C \Delta \\
& +C \sup _{t \in\left[0, h_{n}\right)} \mathbf{E}\left\|X_{0 n}(t, \omega)-x\right\|^{2}
\end{aligned}
$$

Учитывая вид $\widetilde{\delta}$ и $\Delta$, окончательно приходим к утверждению теоремы.

Следствие 3. IIусть $\sigma^{i j} \in C_{B}^{2}\left(\mathbf{R}^{d}\right), a^{i} \in C_{B}^{1}\left(\mathbf{R}^{d}\right), i=\overline{1, d}, j=\overline{1, r}$, $u n \rightarrow \infty, h_{n} \rightarrow 0$ max, umo

$$
h_{n}=o\left(n^{-1}\right)
$$




$$
\sup _{t \in\left[0, h_{n}\right)} \mathbf{E}\left\|X_{0 n}(t, \omega)-x\right\|^{2} \longrightarrow 0 .
$$

Tozda

$$
\sup _{t \in T} \mathbf{E}\left\|X(t, \omega)-Y_{n}(t, \omega)\right\|^{2} \longrightarrow 0
$$

где $X(t, \omega)$ и. $X_{n}(t, \omega)$ - решения задач (1.24) и (1.16) соответственно.

3 а м е ч ан и е 4. Несложно видеть, что условие (1.47) эквивалентно тому, что $\widetilde{h}=\left[\left(h_{n}\right)\right] \in S$.

1.4. Смешанный случай. В этом пункте исследуем предельное поведение решений следующей задачи Коши:

$$
\left\{\begin{array}{c}
X_{n}^{i}\left(t+h_{n}, \omega\right)-X_{n}^{i}(t, \omega) \\
=\sigma_{n}^{i 1}\left(X_{n}(t, \omega)\right)\left[B_{n}^{1}\left(t+h_{n}, \omega\right)-B_{n}^{1}(t, \omega)\right] \\
\quad+\sigma_{n}^{i 2}\left(X_{n}(t, \omega)\right)\left[B_{n}^{2}\left(t+h_{n}, \omega\right)-B_{n}^{2}(t, \omega)\right] \\
\quad+a_{n}^{i}\left(X_{n}(t, \omega)\right) h_{n}, \quad t \in T \\
\left.X_{n}^{i}\right|_{\left[0, h_{n}\right)}=X_{0 n}^{i}(t, \omega), \quad i=1,2,
\end{array}\right.
$$

где $B_{n}(t, \omega)=\left(B_{n}^{1}(t, \omega), B_{n}^{2}(t, \omega)\right), X_{0 n}=\left(X_{0 n}^{1}, X_{0 n}^{2}\right), B_{n}^{1}(t, \omega)=$ $\left(B^{1} * \rho_{n}\right)(t, \omega), \rho_{n}$ определено в $(1.2), B_{n}^{2}(t, \omega)=\left(B^{2} * \rho_{n}^{1}\right)(t, \omega), \rho_{n}^{1}(t)=$ $\varphi(n) \rho(\varphi(n) t), \varphi: \mathbf{R}_{+} \rightarrow \mathbf{R}_{+}-$монотонно неубываюшая функция, а $\rho-$ из соотношений $(1.2), B=\left(B^{1}, B^{2}\right)$ - двумерный стандартный процесс $\mathscr{F}_{t}$-броуновского движения; $\sigma_{n}^{i j}, a_{n}^{i}$ задаются так же, как и в $(1.2)$, для $i, j=1,2$.

Пусть

$$
X(t, \omega)=\left(X^{1}(t, \omega), X^{2}(t, \omega)\right)
$$

- решение системы стохастических дифференциальных уравнений

$$
\begin{aligned}
X^{i}(t, \omega)= & x^{i}+(S) \int_{0}^{t} \sigma^{i 1}(X(s, \omega)) d B^{1}(s, \omega) \\
& +(I) \int_{0}^{t} \sigma^{i 2}(X(s, \omega)) d B^{2}(s, \omega)+\int_{0}^{t} a^{i}(X(s, \omega)) d s
\end{aligned}
$$

$i=1,2, t \in T, x=\left(x^{1}, x^{2}\right) \in \mathbf{R}^{2}$.

Teорема 5. Пусть $\sigma^{i j} \in C_{B}^{2}\left(\mathbf{R}^{2}\right), a^{i} \in C_{B}^{1}\left(\mathbf{R}^{2}\right), i, j=1,2$. Тогдa

$$
\begin{gathered}
\sup _{t \in T} \mathbf{E}\left\|X(t, \omega)-X_{n}(t, \omega)\right\|^{2} \leqslant C \sup _{t \in\left[0, h_{n}\right)} \mathbf{E}\left\|x-X_{0 n}(t, \omega)\right\|^{2} \\
+C n^{2} h_{n}^{2}+\frac{C}{\varphi(n) h_{n}}+\frac{C}{n^{8 / 7} h_{n}}+C n^{-6 / 7+\alpha}
\end{gathered}
$$

где $\|\cdot\|-$ евклидова иорма $ө \mathbf{R}^{2}, X_{n}(t, \omega), t \in T, \omega \in \Omega, n \in \mathbf{N}$, - 
решение задачи Коши (1.48), а $\alpha$ - сколь угодно малое положительное число, $h_{n}<1 / n, 1 / \varphi(n)<h_{n}$.

Доказательство теоремы 5 проводится методами доказательства теорем 2 и 4.

\section{2. Ассоциированные в смысле Ито и Стратоновича решения уравнений в дифференциалах в прямом произведении алгебр обобщенных случайных процессов}

В прямом произведении алгебр $\mathscr{G}(\tilde{T}, \Omega)^{d}\left(\mathscr{G}_{L}(\tilde{T}, \Omega)^{d}\right)$ рассмотрим следующую задачу Коши:

$$
\left\{\begin{array}{l}
d_{\tilde{h}} \widetilde{X}^{i}(\tilde{t}, \omega)=\sum_{j=1}^{r} \tilde{\sigma}^{i j}(\tilde{X}(\tilde{t}, \omega)) d_{\tilde{h}} \widetilde{B}^{j}(\tilde{t}, \omega)+\tilde{a}^{i}(\tilde{X}(\tilde{t}, \omega)) d_{\tilde{h}} \tilde{t}, \\
\left.\tilde{X}^{i}\right|_{[\tilde{0}, \tilde{h})}=\tilde{X}_{0}^{i}(\tilde{t}, \omega), \quad i=\overline{1, d}
\end{array}\right.
$$

где $\widetilde{\sigma}^{i j}=\left[\left(\sigma_{n}^{i j}\right)\right] \in \mathscr{G}\left(\widetilde{\mathbf{R}}^{d}\right), \widetilde{B}^{j}=\left[\left(B_{n}^{j}\right)\right] \in \mathscr{G}(\widetilde{T}, \Omega)\left(\mathscr{G}_{L}(\widetilde{T}, \Omega)\right)$ - обобщенные случайные процессы броуновского движения, $\widetilde{a}^{i}=\left[\left(a_{n}^{i}\right)\right] \in \mathscr{G}\left(\widetilde{\mathbf{R}}^{d}\right)$, $i=\overline{1, d}, j=\overline{1, r} ; \tilde{t}=[(t)] \in \widetilde{T}, \tilde{h}=\left[\left(h_{n}\right)\right] \in H, \tilde{t}+\widetilde{h} \in \widetilde{T}, \widetilde{X}_{0}^{i}=\left[\left(X_{0 n}^{i}\right)\right] \in$ $\mathscr{G}(\widetilde{T}, \Omega)\left(\mathscr{G}_{L}(\widetilde{T}, \Omega)\right), i=\overline{1, d}, \widetilde{X}=\left(\widetilde{X}^{1}, \ldots, \widetilde{X}^{d}\right), \widetilde{X}_{0}=\left(\widetilde{X}_{0}^{1}, \ldots, \widetilde{X}_{0}^{d}\right)$.

В этом параграфе исследуем предельное при $n \rightarrow \infty, h_{n} \rightarrow 0$ поведение решений задачи (2.1) в прямом произведении алгебр $\mathscr{G}(\widetilde{T}, \Omega)^{d}$ $\left(\mathscr{G}_{L}(\widetilde{T}, \Omega)^{d}\right)$.

\section{1. Теоремы существования и единственности.}

Теорема 6. Для того чтобы в прямом произведении алгебр $\mathscr{G}(\widetilde{T}, \Omega)^{d}$ решение задачи Коши (2.1) существовало и было единственным, необходимо и достаточно, чтобы для любых представителей $\left(\sigma_{n}^{i j}\right),\left(a_{n}^{i}\right),\left(X_{0 n}^{i}\right),\left(B_{n}^{j}\right),\left(h_{n}\right), i=\overline{1, d}, j=\overline{1, r}$, выполнялись следующие условия:

$$
\begin{aligned}
& \frac{d^{l}}{d t^{l}} X_{0 n}^{i}\left(h_{n}-s, \omega\right)-\frac{d^{l}}{d t^{l}} X_{0 n}^{i}(s, \omega) \\
& \quad-\sum_{j=1}^{r} \frac{d^{l}}{d t^{l}}\left\{\sigma_{n}^{i j}\left(X_{0 n}(s, \omega)\right)\left[B_{n}^{j}\left(h_{n}+s, \omega\right)-B_{n}^{j}(s, \omega)\right]\right\} \\
& \quad-\frac{d^{l}}{d t^{l}} a_{n}^{i}\left(X_{0 n}(s, \omega)\right) h_{n} \underset{s \rightarrow+0}{\longrightarrow} 0, \quad i=\overline{1, d}
\end{aligned}
$$

для почти всех $\omega \in \Omega$ ияюбых $l=0,1,2, \ldots$.

Здесь $\left(d^{l} / d t^{l}\right) X(t, \omega), l=1,2, \ldots$, есть $l$-я производная случайного процесса $X(t, \omega)$ в смысле сходимости почти всюду по $\omega \in \Omega$. 
Теорема 7. Пусть для любых представителей $\left(\sigma_{n}^{i j}\right),\left(a_{n}^{i}\right),\left(X_{0 n}^{i}\right)$, $\left(B_{n}^{j}\right),\left(h_{n}\right), i=\overline{1, d}, j=\overline{1, r}$, выполняются условия

$$
\begin{gathered}
\frac{\partial^{|\alpha|}}{\partial^{\alpha_{1}} x_{1} \partial^{\alpha_{2}} x_{2} \cdots \partial^{\alpha_{d}} x_{d}} \sigma_{n}^{i j} \in C_{B}\left(\mathbf{R}^{d}\right), \\
\frac{\partial^{|\alpha|}}{\partial^{\alpha_{1}} x_{1} \partial^{\alpha_{2}} x_{2} \cdot \cdots \partial^{\alpha_{d}} x_{d}} a_{n}^{i} \in C_{B}\left(\mathbf{R}^{d}\right)
\end{gathered}
$$

для всех мультииндехсов $\alpha=\left(\alpha_{1}, \ldots, \alpha_{d}\right),|\alpha|=\alpha_{1}+\cdots+\alpha_{d}, \quad u$

$$
\begin{aligned}
\mathbf{E}\left\{\frac{d^{l}}{d t^{l}} X_{0 n}^{i}\left(h_{s}-s, \omega\right)-\frac{d^{l}}{d t^{l}} X_{0 n}^{i}(s, \omega)\right. \\
-\sum_{j=1}^{r} \frac{d^{l}}{d t^{l}}\left(\sigma_{n}^{i j}\left(X_{0 n}(s, \omega)\right)\left[B_{n}^{j}\left(h_{s}+s, \omega\right)-B_{n}^{j}(s, \omega)\right]\right) \\
\left.\quad-\frac{d^{l}}{d t^{l}} a_{n}^{i}\left(X_{0 n}(s, \omega)\right) h_{n}\right\}^{p} \underset{s \rightarrow+0}{\longrightarrow} 0, \quad i=\overline{1, d}, p \geqslant 1,
\end{aligned}
$$

для любых $l=0,1,2 \ldots$. Тогда решение задачи Коши (2.1) в прямом произведении алгебр $\mathscr{G}_{L}(\widetilde{T}, \Omega)^{d}$ существует и единственно.

Здесь $\left(d^{l} / d t^{l}\right) X(t, \omega), l=1,2, \ldots$, есть $l$-я производная случайного процесса $X(t, \omega)$ в смысле $L^{p}(\Omega, \mathscr{A}, \mathbf{P}), p \geqslant 1$.

Доказательства теорем 6 и 7 проводятся методом математической индукции.

2.2. Ассоциированные решения уравнений в дифференциалах в $\mathscr{G}_{L}(\widetilde{T}, \Omega)^{d}$. Пусть $\widetilde{X}(t, \omega)=\left[\left(X_{n}(t, \omega)\right)\right]-$ решение задачи Коши (2.1). Случайный процесс $X(t, \omega), t \in T, \omega \in \Omega$, назовем ассоциированным в смысле Ито (Стратоновича) решением данной задачи, если при $n \rightarrow \infty, h_{n} \rightarrow 0$ так, что $\tilde{h} \in I(\widetilde{h} \in S)$, для любого $\left(X_{n}\right) \in \widetilde{X} X_{n}(t, \omega)$ сходится к $X(t, \omega)$ в $L^{2}(\Omega, \mathscr{A}, \mathbf{P})$ или почти всюду по $\omega \in \Omega$ и равномерно по $t \in T$.

Из следствия 2 и теоремы 7 вытекает

Теорема 8. Пусть выполняются условия (1.23), (2.2)-(2.4) и обобщенный случайный прочесс $\widetilde{X}_{0}$ aсcoчиирует $x \in \mathbf{R}^{d}$. Тогда ассочиированное в смысле Ито решение задачи Кочи (2.1) в прямом произведении алгебр обобщенных случайных прочессов $\mathscr{G}_{L}(\widetilde{T}, \Omega)^{d}$ является решением стохастического дифференциального уравнения (1.1).

Из следствия 3 и теоремы 7 вытекает

Теорема 9. Пусть выполняются условия (1.47), (2.2)-(2.4) и обобиенный случайный прочесс $\widetilde{X}_{0}$ accoчиирует $x \in \mathbf{R}^{d}$. Тогда ассочиированное в смысле Стратоновича решение задачи Коши (2.1) в прямом произведении алгебр обобщенных случайных прочессов $\mathscr{G}_{L}(\widetilde{T}, \Omega)^{d}$ является решением стохастического дифференциального уравнения (1.24). 
В теоремах 8 и 9 в качестве обобщенных случайных процессов броуновского движения рассматриваются обобщенные случайные прогессы вида (1.2).

Аналогичные результаты справедливы и в прямом произведении алгебр обобщенных случайных процессов $\mathscr{G}(\widetilde{T}, \Omega)^{d}$.

\section{СПИСОК ЛИТЕРАТУРЫ}

1. Wong E., Zakai $M$. On the convergence of ordinary integrals to stochastic integrals. Ann. Math. Statist., 1965, v. 36, № 5, p. 1560-1564.

2. Sussman $H$. $J$. On the gap between deterministic and stochastic ordinary differential equations. - Ann. Probab., 1978, v. 6, № 1, p. 19+41.

3. Мачкявичюс $B$. $S^{p}$-устойчивость решений симметрических стохастических дифференциальных уравнений. - Литов. матем. сб., 1985, т. 25, в. 4, с. 72-84.

4. Юрачковский $A$. П. Предельная теорема для стохастических разностных схем без запаздывания. - Докл. АН УССР, 1986, сер. А, № 7, с. 24-26.

5. Карабаш $M$. А. Об аппроксимациях решений стохастических уравнений с монотонными коэффициентами. - Теория случайных процессов (Киев), 1986, № 14, c. $25-28$.

6. Гихман И. И., Скороход А. В. Стохастические дифференциальные уравнения. Киев: Наукова думка, 1968, 354 с.

7. Itô $K$., Nigio $M$. On stationary solutions of a stochastic differential equation. - J. Math. Kyoto Univ., 1964, v. 4, № 1, p. 1-75.

8. Maruyama $G$. Continuous Markov processes and stochastic equations. - Rend. Circ. Mat. Palermo, 1955, v. 4, № 2, p. 48-90.

9. Мацкявичюс $B$. Некоторые аппроксимации стохастических интегралов и решений стохастических дифференциальных уравнений. - Литов. матем. сб., 1978, T. 18 , № 3 , c. 101-108.

10. Хорстхемке В., Лефевр $P$. Индуцированные шумом переходы. М.: Мир, 1987, $400 \mathrm{c}$.

11. Лазакович Н. В., Стачуленок С. ПI. Аппроксимация стохастических интегралов Ито и Стратоновича элементами прямого произведения алгебр обобщенных случайных процессов. - Теория вероятн. и ее примен., 1996, т. 41, в. 4, с. 785-809.

12. Егоров Ю. В. К теории обобценных функций. - Успехи матем. наук, 1990, т. 45, B. 5, c. 3-40.

13. Ватанабэ С., Икэда $Н$. Стохастические дифференциальные уравнения и диффузионные процессы. М.: Наука, 1986, 448 с.

14. Рождественский Б. Л. Новый метод решения задачи Коши в целом для квазилинейных уравнений. - Докл. АН СССР, 1961, т. 138, № 2, с. 309-312.

15. Кузмечов $H$. H. О применении метода сглаживания к некоторым системам гиперболических квазилинейных уравнений. - Журн. вычисл. матем. и матем. физики, 1973 , т. 13, № 1, с. 92-102. 